\title{
LA INSTITUCIÓN UNIVERSITARIA EN LA GLOBALIZACIÓN NEOLIBERAL: CONSIDERACIONES DESDE UNA PERSPECTIVA DE LA GESTIÓN UNIVERSITARIA
}

\author{
ALEJANDRO ELIAS OCHOA-ARIAS \\ Universidad de Los Andes, Venezuela \\ Universidad Austral de Chile, Puerto Montt, Chile \\ Jesús LARA-CoRONADO \\ Universidad Austral de Chile, Puerto Montt, Chile
}

\begin{abstract}
Resumo: Se presenta un modelo de gestión universitaria de inspiración neoliberal, propia del discurso hegemónico globalizante, para dar cuenta de lo que es el proceso de construcción de la universidad chilena del presente. El modelo de gestión universitaria descansa sobre los tres ejes considerados estratégicos en la universidad contemporánea y privilegia la gestión desde una racionalidad neoliberal que subyace a los procesos organizacionales, en particular la evaluación y acreditación.
\end{abstract}

Palavras-chave: Racionalidad. Neoliberalismo. Universidad. Chile.

\section{LA UNIVERSIDAD COMO ESPACIO DE DISPUTA}

La consideración de la educación como espacio de disputa política y, en especial, en lo que concierne a la institución universitaria es uno de los resultados más notables en los procesos sociales que vive América latina desde hace ya varias décadas. Los conflictos están estrechamente conectados con el rol de la universidad como catalizador de los procesos de transformación popular o, en su contraparte, como mecanismo para la formación técnica de los cuadros encargados de la conducción aséptica de los procesos productivos de la sociedad propios de los procesos de globalización que tiene como marco racional el asociado al neoliberalismo. El aparato educativo queda así expuesto en la cúspide de los procesos de formación, en un debate que no ha abandonado a la universidad latinoamericana desde que irrumpe la universidad republicana. Sin embargo, la evolución y desplazamiento de las formas productivas hegemónicas que se centran ahora en el conocimiento como mercancía, ponen sobre el debate algo más profundo que la disputa secular de la autonomía universitaria. Se trata de la incorporación de la sociedad en procesos centrados en el cultivo de conocimiento en la cual el énfasis ya no radica en la explotación de los recursos físicos de un determinado territorio ni de los procesos de industrialización sino de la capacidad de innovación y generación de valor a partir del 
conocimiento. El surgimiento del conocimiento en términos del valor de uso en la sociedad contemporánea significa que su producción se podría entender claramente como una cadena productiva con un determinado costo y beneficio. El debate que antes suponía a la universidad como un espacio de disputa sin mayores transformaciones en su quehacer institucional, es ahora el objeto de transformación pues sus modos de producción del conocimiento, divulgación e investigación son puestos en tensión porque se les cuestiona en términos de una racionalidad con pretensión de universalidad. Una pregunta que mueve este artículo es sobre la relevancia del modelo neoliberal para el proceso de transformación organizacional de la universidad y su impacto en los procesos de globalización. Esta relevancia será considerada tomando como manifestación concreta a la institución universitaria chilena dado que es precisamente el modelo chileno de sociedad, ejemplo de globalización para la sociedad contemporánea latinoamericana.

La universidad en términos de su tránsito histórico ha estado signada por la búsqueda del conocimiento anclado en la referencia de un orden del mundo que se busca revelar y comprender (MacIntyre, 2009). De aquel propósito que acertadamente se señala como una institución construida a imagen y semejanza de un orden cósmico (Maclntyre,2009) se ha devenido en un espacio en el cual se abren paso los modos de enseñar y formar técnicamente que sugieren a la universidad como espacio de entrenamiento y formación profesional. Esta transformación de la universidad ha ocurrido a lo largo del tiempo en una disputa donde la resistencia a sucumbir a la búsqueda de modos de ordenar el mundo para la producción exitosa y el desarrollo de los circuitos tecnológicos de transformación de la naturaleza, al ser humano y la sociedad no ha sido particularmente exitosa. La existencia de una universidad comprometida con la crítica y otra con la producción material paulatinamente va constituyéndose en uno solo que es el gobernado por la lógica ya no de las ciencias sino de las técnicas. La confrontación entre la lógica de ciencias sociales y naturales que dominó el debate epistemológico hasta finales del siglo pasado (Habermas, 1990) se fue paulatinamente desdibujando en el ámbito de las instituciones universitarias por una lógica de la producción científica, la visibilidad y la aplicación del conocimiento. La aparición de la bibliometría, cienciometría y webmetria pueden ser consideradas como métricas que son externas a las prácticas de la disciplina y que apuntan en la dirección de medir cuantitativamente al conocimiento en tanto que resultado de una cadena valor. El impacto de estas métricas en la configuración organizacional de la universidad nos parece un ámbito relevante de estudio que no atenderemos en este artículo.

¿Qué ocurre en el ámbito latinoamericano con respecto al discurso globalizador, el pensamiento neoliberal y la universidad? Una posibilidad es que el neoliberalismo constituye el referente político-ideológico sobre el cual las transformaciones organizacionales de la universidad se adecúan al modelo de globalización hegemónico. El que existan realidades tan diversas en un mismo continente donde la gratuidad de la educación y la educación como servicio del cual se obtiene lucro puedan convivir, lejos de mostrar la existencia de realidades separadas, muestra el espacio de disputa que consigue en cada sociedad sus propias formas de conflicto y diferencia. El que podamos interrogarnos sobre la universidad fundada en el neoliberalismo, arrojará algunas luces sobre lo que se puede esperar en la construcción organizacional de la universidad como 
espacio globalizado y los límites de tal construcción, en la sociedad latinoamericana máxime cuando el discurso organizacional está cada vez más anclado en una racionalidad instrumental económico-céntrica considera como la única posible para la globalización.

\section{CONSIDERACIÓN METODOLÓGICA}

Este artículo se construye a partir de dos propósitos diferentes pero complementarios. Por una parte, se trata de escudriñar sobre los modos de organización que serían propios de un modelo neoliberal de sociedad considerado propicio para los procesos de globalización en el cual se inserta la universidad. Para ello apelaremos a la formulación de un contexto interpretativo anclado en una lectura del neoliberalismo centrada en Brown (2003). Desde esa plataforma, formular lo que sería un tipo-ideal de la universidad y utilizarlo para dar cuenta de un corpus construido desde el ámbito de la historia de la educación contemporánea en Chile.

El modo de construcción de este modelo ideal privilegiará el tema de la gestión universitaria como institución. Para ello, se identifican los conceptos claves de una globalización de condición neoliberal a partir de los cuales se elabora un modelo básico de las actividades estratégicas de la gestión universitaria.

Aunque no se apela en el objeto de estudio a una forma institucional empírica se abordará lo que corresponde a discursos asociados al ámbito universitario en relación con la historia más reciente de la universidad chilena y alguna revisión de los procesos de construcción de sujetos universitarios en la globalización neoliberal.

\section{CONTEXTO DE LA GESTIÓN NEOLIBERAL DE LA UNIVERSIDAD EN LA GLOBALIZACIÓN}

Antes de caracterizar lo que serían las actividades asociadas a la gestión universitaria y lo que consideraríamos como sus actividades medulares fundamentales: formación profesional, investigación-desarrollo y la extensión universitaria, es necesario esbozar lo que serían los fundamentos de este modelo de gestión. Para ello, apelaremos a una concepción neoliberal de la globalización asumiendo la concepción del neoliberalismo en los términos desarrollados por Wendy Brown (2005) porque provee una interesante trayectoria para mostrar el proceso de construcción del neoliberalismo de una forma sintetizada y con el centro en el pensamiento de Foucault, que permite dar cuenta del neoliberalismo desde una postura más comprensiva que aquella expuesta en términos esencialmente económicos.

La globalización ha respondido esencialmente a procesos de apertura de mercados nacionales y la homologación de los criterios con los cuales se evalúan los procesos de intercambio para garantizar una transparencia en esos procesos. Es decir, la puesta en marcha de una racionalidad que privilegie las "buenas prácticas" más allá de las consideraciones que son propias a los contextos socio-culturales en los cuales son inevitables las diferencias, va propiciando una noción de lo "bueno" que se asocia a lo rentable. La imposición de la cuantificación económica como forma "universal" deviene en la manifestación de una racionalidad que se supone global. ¿Cuáles son las ventajas y desventajas de globalizar una racionalidad? 


\section{EL NEOLIBERALISMO COMO RACIONALIDAD}

El neoliberalismo puesto en una idea generatriz será entendido en este trabajo como la «diseminación y extensión de los valores del mercado a todas las instituciones y la acción social» (Brown, 2005). Lo distintivo con respecto al discurso más difundido del neoliberalismo es en asumir que las medidas de racionalidad y coherencia en todas las instituciones y la acción social serán los valores del mercado. Términos como utilidad, eficiencia en costos, competencia y oportunidad serán los que proveerán las bases para evaluar todas las instituciones y cada uno de los procesos que en ella se desarrollan. Todo deviene en una empresa que se gesta desde la individualidad de cada miembro de la organización y que se va expandiendo a lo largo de la cadena de procesos en las cuales los criterios que regulan esa cadena sean los propios del mercado global imperante.

La consideración "genética» del neoliberalismo así entendido, entonces tendrá sus manifestaciones emergentes en lo que siempre se han considerado como sus metas: un mercado siempre creciente, la ausencia del estado en funciones de regulación de los efectos del mercado y la ausencia de mecanismos de compensación. Se trata de un proceso de globalización de lo que serían las "buenas prácticas" de gobierno porque son las prácticas que se pueden hacer comunes. Es la instauración de reconocer una condición de riesgo que es necesaria para crear las condiciones de desigualdad que demanda un proceso competitivo que ahora adquiere un status global. Instaurado desde la propia racionalidad con la cual el individuo concibe su participación en una actividad social como una relación gobernada por el mercado, entonces el neoliberalismo que interesa mostrar es aquel que se refiere a estos mecanismos «ocultos» en los cuales la organización de procesos va a estar concentrada en la realización de una eficiencia económica.

El dominio de esta racionalidad comporta para el espacio de las acciones concertadas de los individuos para alcanzar un determinado fin, el que este fin sea traducido en términos económicos porque esa es la razón última de la justificación de la acción concertada. En el contexto organizacional, el entorno se convierte esencialmente en espacio de competencia y de cálculo de oportunidades para incrementar los beneficios en términos económicos. El que esto sea así, implica que la evaluación del desempeño de cualquier organización responda a una métrica acordada en términos económicos lo que impone la conversión de las distintas dimensiones en las cuales una organización hace presencia en la sociedad en términos estrictamente económicos. Este proceso de conversión de todos los bienes en términos económicos supone una gestión que pueda desarrollar la capacidad de hacer esa traducción y, posteriormente, proceder al análisis del desempeño económico. La transparencia derivada de la exactitud en las cuentas no da cuenta del proceso de traducción que le antecede y de las concesiones que son necesarias para alcanzar un espacio mensurable.

El modo de gestión estará entonces caracterizado por la economización de todos los procesos y la reducción de las fuentes de incertidumbre que pudieran devenir en costos ocultos o pérdida de los márgenes de ganancia. La reducción del riesgo será construida por la instauración de procesos homogéneos y el desarrollo de medidas que permitan que el proceso de intercambio con el resto de los actores de la sociedad pueda estar regulado por la razón económica. La organización con gestión sobre estos criterios 
estará centrada en el desarrollo del capital que define o condiciona el valor social de la organización en términos de los bienes o servicios que ella brinda a la sociedad. En el proceso de globalización, no sólo será necesario tener acceso al mercado global sino además, poder expresarse en los términos de esa racionalidad omnipresente de la economía. A continuación, se muestran algunas consecuencias de estas consideraciones para la gestión universitaria.

\section{GESTIÓN NEOLIBERAL DE LA UNIVERSIDAD GLOBAL}

Una premisa sobre la cual se desarrolla este contexto de sentido sobre la gestión universitaria estriba en la condición histórica de la institución universitaria. Por ello, no nos referimos a la gestión de una universidad neoliberal, sino la gestión neoliberal de la universidad en el supuesto que las universidades en el entorno latinoamericano han estado influenciadas por diversas fuerzas que han condicionado su gestión, desarrollo y modernización. Entre estas fuerzas nos interesa rescatar aquellas asociadas a los procesos de la autonomía universitaria que marca la historia de la universidad latinoamericana con la reforma de Córdoba como referencia de una universidad de compromiso con lo autóctono y popular. Por otra parte, la reciente tendencia de la constitución del conocimiento como motor de la economía (la sociedad del conocimiento) que ubica a la universidad como actor clave en los procesos de transformación económica y como un espacio privilegiado de la globalización.

¿Cuáles serían las implicaciones de una racionalidad neoliberal en la gestión universitaria? La primera respuesta es que el núcleo de toda acción no sería el cálculo egocéntrico (los intereses de cada individuo) sino un cálculo económico. De allí que toda acción con intención deberá entenderse/traducirse como una acción económica. El criterio de medida entonces será el que pueda reportar un beneficio cuantificable en el mercado. Nótese que esto implica no sólo lo cuantitativo que pudiera entenderse con lo medible que es propio de toda gestión, sino que eso medible debe ser tasado y apreciado por el mercado. ¿Cuáles son las características propias del mercado para la universidad? El mercado de la universidad puede constituirse por tres instancias directas: a) Un mercado definido por los procesos de formación de capital humano; b) El mercado asociado a la investigación e innovación y, c) el mercado constituido por los programas de extensión. Estos mercados están asociados a los procesos de compra/venta de servicios en un área determinada del conocimiento lo que implica que para cada mercado deberá identificarse las características de los clientes en términos de optimización de lo económico. Es la búsqueda de una homogeneidad del cliente para asegurar la provisión del servicio y, por otra parte, poder atender las demandas específicas del cliente. Esta demanda supone no sólo una continua actualización del servicio, implica además la versatilidad del servicio para adecuarse a la demanda de cada individuo. Veamos cada uno de esos mercados.

\subsection{FORMACIÓN DE CAPITAL HUMANO}

El mercado de formación de capital humano se concibe como una espiral que inicia con la provisión de un servicio que garantice las competencias mínimas para el éxito del individuo que las adquiere y que corresponde a las estipuladas por el mercado laboral. El servicio no responde a la demanda específica de cada cliente directo, sino que se 
adecúa al perfil que se construye desde el mercado laboral. La universidad es parte de una cadena de valor del capital cognitivo en la cual ella es proveedora tanto a un mercado constituido por las organizaciones que definen competencias y las demanda en términos generales y la del mercado de quien asume la adquisición de esas competencias para ser económicamente atractivo para el mercado. La condición de producto y cliente se resume en un mismo sujeto. La demanda se irá individualizando entre lo que es una contraparte que es capaz de ir delineando su condición de cliente en cuanto a sus aspiraciones y el modo de satisfacerlas, es decir, se alcanza un grado máximo de "libertad" que radica en la capacidad de autoservicio para poder elevar el capital cognitivo del sujeto/producto para un intercambio económico beneficioso en el mercado laboral. Es en su versión más elaborada una "máquina de auto-construcción de competencias" para la satisfacción del mercado. La preservación e incremento del capital cognitivo de cada individuo es una tarea individual que demanda de este un continuo actualizarse en términos de competencias que sean atractivas para el mercado. Este proceso de actualización de competencias incorpora al aparato universitario en una cadena de valor económico que tiene dos características que son propicias para el rendimiento económico de la actividad. Uno es la continua necesidad de actualización que genera un mercado cautivo en los clientes individuales y una vinculación estrecha con los sectores corporativos para establecer mecanismos eficientes de actualización y validación de las competencias por demandar. Esto sin considerar el impacto que tiene que el académico es quizás el sujeto/producto por excelencia de este circuito (Casanova y Boitia, 2019).

\subsection{INVESTIGACIÓN E INNOVACIÓN}

El proceso de investigación ocupa un lugar importante porque es el eje capaz de generar lo que se considera como el bien propio de la universidad: conocimiento. Acá el concepto de cliente experimenta una explosión por las múltiples dimensiones en las cuales ofertar sus servicios. Por una parte, se cuenta con un mercado directo constituido por aquellos quienes financian la investigación y cuyo producto final es el objeto de transacción. Es de algún modo la puesta de la universidad al servicio de otra organización en el servicio de investigación y desarrollo. En una segunda instancia, están los otros mercados (académicos, innovadores, estudiantes) que adquieren en el producto (producto acabado, forma de producirlo, subproductos, incluso errores en la elaboración) nuevas competencias, posibilidades de innovación y de generar nuevos productos finales. Esta modalidad de mercado reporta beneficios en escalas de tiempo distintos. Finalmente, en última instancia aparece el ofrecimiento del producto o servicios en el mercado a partir de los procesos de innovación. Este último aspecto juega un papel estratégico importante porque constituye la continuidad y usufructo de servicios/productos a lo largo del tiempo. El que la innovación ocupe un lugar importante en los procesos de la universidad es señal de la apropiación de la universidad de los procesos de prolongación de la relación de servicio con los consumidores/clientes de sus productos. Este proceso de explosión de clientes y de modalidades de intercambio ha encontrado en los procesos de propiedad intelectual un mecanismo de control que permite preservar la propiedad incluso de aquello que en este momento no tenga claramente un valor de mercado. El entramado así dispuesto finalmente logra ejercer un 
poder "invisible" incluso sobre quien se supone ha hecho ejercicio de su libertad: el investigador. En el circuito del mercado, el investigador es producto, cliente y proveedor. Es en este espacio donde el capital cognitivo de cada investigador juega un rol importante en la preservación y continuidad de la cadena valor que sea capaz de desarrollar con su entorno (Torres y Schugurensky, 2002).

\subsection{VINCULACIÓN CON EL MEDIO}

Este mercado se caracteriza por ser marginal a los otros previamente identificados. Su condición de marginal se refiere a la naturaleza del servicio que reporta que bien pudiera considerarse como el resultado de la holgura productiva en los dos renglones anteriores. No obstante, por la propia evolución histórica de la universidad, este puede constituirse en el espacio en el cual aquellas disciplinas o áreas del conocimiento que no hayan podido generar cadenas valor con desempeño satisfactorio, se ocupan de atender servicios que pueden ser de publicidad y divulgación de la universidad con el propósito de dirigirse a un mercado más amplio y que potencialmente podría formar parte de los mercados cautivos identificados anteriormente.

La particularidad de este mercado estriba en acceso a recursos económicos que no están directamente vinculados con las actividades primarias de la universidad $y_{\text {, }}$ curiosamente, apela a un reducto en el cual la racionalidad económica no parece funcionar con facilidad, pues tiene que ver con aspectos como prestigio, legitimidad que aunque pueden tener su correlato en términos de ganancias económicas, queda en evidencia la necesidad de una traducción de una dimensión a la otra. Esta condición comienza a mostrar que incluso en el caso de un entorno definido en términos del mercado es necesario contar con mecanismos de traducción. Un papel importante en este proceso de traducción lo juegan la definición de los rankings asociados a prácticas que están definidos en términos de cuantificación que son aceptados ampliamente, incluso más allá del entorno inmediato e incluso, minimizando su impacto.

\subsection{GESTIÓN ORGANIZACIONAL}

El entramado organizacional estaría concentrado en ser eficiente en la realización de los beneficios económicos que reportan las actividades identificadas como primarias de la universidad. Toda operación universitaria requiere ser justificada en términos económicos y requerirá de la experticia que aporte la métrica correspondiente. Esto da lugar a la aparición de tareas, procesos e inclusos subsistemas en la organización universitaria que deberán no sólo garantizar el éxito en términos económicos sino además deberán desarrollar las capacidades para poder traducir el desempeño de la universidad en ambos sentidos. Por una parte, deberán ser capaces de convertir la práctica propia asociada a los procesos de aprendizaje en términos contables y económicos. Por otra parte, deberá justificar los procesos de inversión de capital en la realización de esas actividades. Un aspecto fundamental de este proceso de "traducción" es la reducción de incertidumbre que deberá desarrollarse para justificar la acción de inversión económica. Esta reducción de incertidumbre demandará que los procesos de captura de mercados (en los distintos niveles identificados anteriormente) sean precisamente de forma segura y que garanticen el respectivo retorno en el tiempo programado y sin deteriorar la calidad de los productos ofertados. 
Este constituye quizás el nuevo entramado organizacional sobre el cual se construye ahora la gestión global neoliberal de la universidad: Aseguramiento de la calidad. Pero, ¿Qué significa asegurar la calidad en la universidad? ¿Es posible plantearse la reducción de incertidumbre y garantizar la calidad del producto? ¿Qué tipo de universidad se necesita bajo las condiciones de producción segura de capital cognitivo? La respuesta inmediata a esta pregunta supone un ecosistema organizacional, tecnológico y científico en constante expansión y revisión de las propias fronteras sobre las cuales opera la innovación.

A la luz de lo anterior, es necesario preguntarse si la consideración del entorno en el cual la universidad despliega sus actividades, guiadas por una racionalidad económica, es un factor crítico para el éxito de esta forma de gestión de la universidad. La pregunta abre dos vías posibles de exploración. La primera, es la vinculada con asumir que todo el entorno de la universidad está guiado por la misma racionalidad $y$, en consecuencia, podríamos entender que el comportamiento sistémico asociado a una misma racionalidad sería expandible a todo ese entorno. Sería el dominio absoluto de una racionalidad económica el espacio apropiado para que esta gestión pudiera encontrarse en condiciones privilegiadas, es decir, la globalización como una racionalidad neoliberal. El privilegio fundamental estriba en obviar los procesos de traducción o conversión de la racionalidad (si es que hubiere una racionalidad distinta en las prácticas de generar, enseñar y divulgar conocimiento), pues aquella quedaría limitada a esos espacios y el proceso de conversión sería tarea para el entramado organizacional, social y tecnológico, asimismo tendría la capacidad de realizar en tanto que todo se constituye y concibe como mercado. Ese presupuesto conceptual es el que permitirá considerar para el caso testigo que se presenta en breve.

\section{LA UNIVERSIDAD Y EL ENTORNO LATINOAMERICANO}

La consideración del mercado como factor crítico en la gestión de la universidad latinoamericana es de aparición paulatina en los años 80 con los procesos de privatización masivos experimentados en muchas sociedades en América Latina (Ibarra, 2012). Un caso exitoso lo constituye el chileno pues logra no sólo instaurar una forma de mercado como mecanismo "ordenador" de la sociedad sino de la propia educación (Basso, 2016, p. 22). Es por ello relevante revisar los resultados de la gestión de la universidad chilena en los últimos 30 años.

¿Qué podemos entender por el entorno desde la perspectiva de la universidad latinoamericana? La respuesta tiene abundantes estudios que demuestran los avances y retrocesos experimentados durante el siglo que nos separa desde la reforma de Córdoba que propicia el modelo de universidad latinoamericana anclada en una idea de universidad popular (ver ref. Declaración liminar) y que tuvo diversas formas de desarrollarse en la América Latina del siglo XX hasta la actualidad, pero nos interesa caracterizar el entorno a la luz del modelo neoliberal, para ello deberemos enfocarnos en el modelo de sociedad hegemónico y su impacto en la configuración de este mercado.

El discurso en torno al cual se puede dilucidar el entorno universitario en la sociedad latinoamericana y salvando las diferencias específicas (históricas, geográficas, 
culturales y políticas) las podemos estructurar en tres discursos distintos en el siglo XX y siglo XXI. La universidad como instrumento de formación ciudadana, la universidad para el desarrollo científico-tecnológico y la universidad para formar profesionales (ver Fuenmayor, Bonucci y López-Garay, 1990). Sin pretender explorar las implicaciones de estos discursos, ellos revelan el papel que se le ha adjudicado a la universidad desde la instauración republicana hasta comienzos del proceso de globalización que demanda una conversión de la universidad como mecanismo de modernización política y económica para convertirse en engranaje para la integración del aparato productivo de la sociedad al orden global. ¿Cómo se ha manifestado esto en el caso concreto de Chile?

La razón de escoger a Chile como testigo del modelo típico-ideal propuesto tiene que ver con encarnar en el caso de América Latina, la sociedad con mayores avances en la instauración de un modelo global neoliberal que permitirá mostrar lo que significa la universidad en este orden global en el contexto latinoamericano.

\section{UNIVERSIDAD ESTERILIZADA POR LA CRECIENTE GLOBALIZACIÓN. CASO CHILE:}

El modelo de universidad heredado del siglo XX en Latinoamérica adquiere una configuración propia en Chile. La visión de cómo se debía dar esta relación, en el contexto chileno, se introdujo con fuerza y por la fuerza a mediados de los años 80 del siglo pasado y sin ningún tipo de resistencia de las casas de estudios, pues el contexto social y político no permitía la disidencia, por lo tanto, las universidades comenzaron a experimentar y a entender que, bajo esta lógica impuesta, la del financiamiento de las universidades, para subsistir necesitaban consumidores no estudiantes. Por esta razón, como estrategia de mercado, para atraer a futuros consumidores se crearon los créditos universitarios, que servían como paraguas para todas aquellas familias que no tenían la solvencia económica para sostener a un integrante de su familia en la educación superior. Aunque este medio para acceder a la universidad no fue ofrecido a los estudiantes mostrando todo lo que conllevaba adquirirlo, por ejemplo; las altas tasas aplicadas a los créditos que significaron un alto endeudamiento en quienes los usaban (Pressaco \& Carbone, 2010, p. 543), sobre todo en quienes no culminaron nunca sus estudios. El que esto ocurriera revela la insuficiencia en el nivel de comprensión de la realidad que gobierna a la universidad por parte de sus clientes/productos y, lamentablemente, sus desechos.

Esta situación explica porque las casas de estudios comenzaron a dedicar sus esfuerzos, por medio de la oferta educativa, a configurar un mercado que no necesariamente respondiera a necesidades contextuales del país o de la región, sino a los requerimientos estandarizados propuestos por agencias de calidad y de acreditación quienes finalmente se convierten en los mecanismos de promoción y evaluación de las universidades. Por consiguiente, los estudiantes que requerían las universidades chilenas como clientes, inmediatamente se convierten en producto para satisfacer las demandas de la globalización. Aquí los ofrecimientos de calidad, de todas las instituciones, principalmente, las que integraban el Consejo de Rectores de las Universidades Chilenas ( $C R U C H)$, que son las que se tuvieron que reinventar durante y después de la dictadura (1973-1990), sobre todo por el tipo de financiamiento que comenzaron a recibir, jugaron un papel fundamental en la sociedad, ya que eran las más codiciadas por los futuros estudiantes universitarios (Pressaco \& Carbone, 2010, pp. 546-547). 
El Estado, de un modelo estatista-garante pasó a uno en el cual la participación de la iniciativa privada constituye el eje sobre el cual interviene o no el estado. Esto implicó 
que las universidades comenzaron a contemplar en sus proyecciones de oferta educativa el retorno económico, pero cuidando que el producto académico no sufriera detrimento. Esto trajo consigo, que en el mundo donde se declara que producción del conocimiento es su hilo conductor: la universidad, ahora para poder subsistir, debía comenzar a utilizar una racionalidad hegemónica, la cual proponía como único camino al éxito una racionalidad de acumulación del capital económico, y la puesta de lo cognitivo, profesional e intelectual en términos económicos. Además, bajo esta lógica, se construye un discurso único en la toma de decisiones de la sociedad, es decir, un conocimiento autolegitimado y no la discusión del conocimiento (Habermas, 1975). En consecuencia, el modelo de la universidad chilena se concentra en procesos de mercadeo (producción de "eslóganes") que captarán la atención del consumidor educativo por medio de ofertas confeccionadas para aprehender a sus futuros estudiantes o, mejor dicho "compradores", mediante las cuales prometían desarrollar perfiles educativos que responden más a criterios de mercado establecidos por las dinámicas sociales e ideológicas ancladas en la globalización, que a discernimientos locales. Esto trajo consigo que ciertos procesos, sobre todo los que deben responder a la formación profesional del estudiante, sean oscurecidos y no considerados dentro de los argumentos centrales de los proyectos educativos universitarios. Por ejemplo, la poca regulación para la aprobación de carreras que no son necesarias por la alta oferta de profesionales, pero muy populares entre quienes las escogen como proyección profesional.

En este sentido, es preciso mencionar que el surgimiento de carreras nunca estuvo regulado por la necesidad nacional que percibían las instituciones de educación superior, esto tampoco lo regula el Estado. El juego de la oferta y la demanda impone el ofrecer las carreras mientras haya clientes dispuestos a matricularse en alguna de las carreras ofrecidas. No es requerido a las universidades un discurso legitimador como tampoco a las instancias públicas que justifiquen el cultivo de un determinado tipo de conocimiento en función de criterios diferentes a los esbozados por sus consumidores quienes están dispuestos a comprar lo ofrecido para mejorar su futuro: "los intereses económicos de un mercado transnacional de la educación que todo lo estandariza según la productividad y la eficacia pragmática" (Mejía Jiménez, 2011, p. 145). O sea, la existencia de las universidades se sustentó en el costo-beneficio, un planteamiento economicista de la educación, el cual por tratar de igualar u homogeneizar las necesidades o requerimientos educativos de las sociedades, queda reducido al tamaño del mundo de quien impone y no al tamaño de ese pequeño pedazo de mundo donde se ejecuta el proyecto educativo. Para decirlo en términos de la centralidad de la globalización, es el desplazamiento del reconocimiento de lo otro como digno de estudio por la figura de la sujeción a un orden que lo determina en el terreno de los hechos y las cifras económicas. En este sentido, los clientes que se matriculan, son justamente estudiantes con familias que tienen fe ciega en los eslóganes ofrecidos por las instituciones de educación superior y que visualizan en las ofertas universitarias la posibilidad de cambiar su destino económico y social, ya que el: "El sistema educativo, quizá más que cualquier otra institución social contemporánea, se ha convertido en el laboratorio donde se prueban diferentes soluciones a los problemas de liberación personal y la igualdad social" (Bowles; Gintis, 1981, p. 16). Esto indudablemente es una oferta engañosa del mercado educativo 
de la educación superior, frente a la cual las universidades tienen un discurso disfrazado, que oculta una realidad que, contrariamente a lo que cree la sociedad, es totalmente opuesta, pues la mayoría de los educandos que se matriculan en las universidades chilenas, casi la mitad de ellos, no termina de estudiar, por lo tanto, hipotecan sus futuros, sin tener las condiciones económicas para solventar la deuda, "la tasa de titulación de un $45 \%$ es la mejor prueba al fracaso del modelo neoliberal aplicado a la educación superior" (Basso, 2016, p. 33).

Si los pilares fundamentales de las universidades se basan en falsedades, pues es lo que venden, esto implica de alguna manera que las instituciones de educación superior se van quedando sin identidad propia, o con identidad de "mercado", por lo tanto, no son capaces desde su rol de colaboración social con la sociedad, responder de manera recíproca a ese llamado. Por ende, el modelo de universidad en Chile no está enfocado en la resolución o discusión de temas considerados relevantes desde la realidad definida en términos locales o regionales del país, pues las investigaciones o producción del conocimiento, mucho de él emanado desde los contextos universitarios, ayudan más a alimentar y crear formas de pensamiento experto y consolidado a nivel global antes que contextualizarlos y debatir críticamente su pertinencia para la sociedad en la cual la universidad se entiende como un espacio educativo. Espacio educativo que finalmente queda minimizado a un espacio donde "la economía empresarial [adapta] y [desvía] los golpes dirigidos a sus fundamentos" (Bowles; Gintis, 1981, p. 16 los corchetes son nuestros).

Es decir, el sistema educativo se transforma en el espacio en el cual la economía neoliberal, a través de la globalización, entendida como la interconexión de ideologías, políticas y cultura que "trasciende las fronteras nacionales" (Ordorika, 2006, p. 32), comienza a dirigir las proyecciones de su patrimonio comercial e ideológico a la competencia del mercado no nacional, sino internacional impuesto por medio de una realidad virtual. Este mercado no está regulado ni dirigido. Al contrario, lo caracteriza la ausencia de cualquier intención por reflexionar por las necesidades de las comunidades con las cuales se vincula más allá de aquellas definidas en términos económicos, pues "las grandes universidades extienden sus ofertas y concentran para sus programas 0 proyectos la demanda educativa, sin que medien estudios de contexto o de pertinencia regional o local" (Carvajal, 2017, p. 155).

\subsection{LA ACREDITACIÓN COMO HERRAMIENTA ESTANDARIZADORA}

La evaluación, calificación y la difusión de los resultados de las universidades en lo que concierne a sus tres ejes (docencia, investigación y vinculación con el medio) constituyen un espacio crítico a la hora de preservar y crecer en el mercado. Los criterios usualmente son definidos a partir de conceptos comunes, cuantificables y aprobados por el orden global. La promoción de "buenas prácticas" se entienden como prácticas cuantificables y afines al modelo de globalización neoliberal que serán a su vez, el instrumento que asegure la vigencia en el mercado educativo, permitiendo y asegurando la salud financiera de la Universidad con su respectivo impacto en la credibilidad institucional y su prestigio social; aspectos que promueve la captación de clientes. Al respecto, Rodríguez señala ".. en este sistema de educación superior las personas eligen 
lo que pueden y lo que pueden está condicionado, antes que nada, por la cuna en la cual nacieron" (Rodríguez, 2012, p. 131), destacando la preeminencia de lo económico sobre 
cualquier otro criterio para tener acceso a la educación universitaria y para realizar sus propias tareas de planificación de las actividades universitarias. La diseminación de este modo de entender la educación se va convirtiendo además en el fundamento para la definición de las políticas que ahora se articulan en función ya no de intereses de los sectores poderosos de cada sociedad sino en nombre de una racionalidad que se despliega globalmente.

Lo anterior es otro de los problemas que se origina por la falta de regulación del Estado hacia las universidades, lo cual demuestra las contradicciones de las reestructuraciones o reformas educativas implementadas en las últimas décadas, ya que el Estado desde un rol de garante pasó a ocupar uno de subsidiario (Donoso; Alarcón, 2012), pues el verdadero ente regulador de las instituciones de educación superior en Chile surge desde las tendencias universitarias internacionales, las cuales marcan pautas de consumo que se originan por datos levantados por las mismas universidades o por otras instituciones que gozan de credibilidad internacional: UNESCO, Banco Mundial (Ordorika, 2006).

Los datos reunidos para planificar el sistema educativo, sirvieron para tributar a los intereses de las trasnacionales, que por medio de sus lógicas de mercado fueron solicitando a las instituciones de educación superior (también en los otros niveles educativos), el desarrollo en sus educandos de las nuevas habilidades y requerimientos que la industria y la tecnología necesitaban para finales del siglo XX y comienzos del XXI. Un aliado frecuente de esta práctica ha sido la OCDE, la que funciona como la decana de las acreditadoras a nivel mundial, marcando rumbos, caminos, ranking y necesidades educativas en la educación superior, al respecto Bürgi nos comenta lo siguiente (2017):

\begin{abstract}
Surgió toda una diversidad de redes "tecnocráticaseducacionalizadoras", que encontraron en la OCDE la plataforma perfecta para implementar y difundir sus ideas. La interrelación de estas redes y sus "estilos de razonamiento" establecieron y propagaron un tipo específico y no universitario de experiencia educativa; con la idea del mundo como laboratorio, esa experiencia culminó en formas de "ingeniería social" afianzadas principalmente por conceptos, más que científicos, políticos (p. 161).
\end{abstract}

Por ello es tan importante que para que este proceso tenga vitalidad exista una "supervigilancia" orientada a la instauración de los principios de mercado internacionales, por lo tanto, "han hecho de la evaluación y la certificación elementos centrales de las políticas públicas en materia educativa en todo el orbe (Ordorika, 2006, p. 37). Esto explica de alguna manera porque las carreras en Chile, aunque tengan límites de matriculados por año de ingreso, el superar este límite de inscritos no se ve como un elemento negativo, al contrario, desde la economía educativa este un factor positivo, pues indica que los clientes están consumiendo y comprando un producto que les ofrece la oportunidad de mejorar sus futuros y la demanda supera la oferta, elemento de éxito en el mercado. Por ello es normal que haya exceso en la incorporación de estudiantes sin tomar en consideración otras variables no menos importantes como capacidad en infraestructura y personal. Pero lo que sí visualiza esta práctica, la matrícula, es una especie 
de sobreventa que se produce en el mercado educativo y no necesariamente indica que ese excedente producido se invertirá en esa misma carrera o en la misma institución, pero sí indica que esa institución, que tiene sobrecupo de matriculados, le está quitando clientes a su análoga y goza de mejor prestigio, economía y consumidores. Es decir, la articulación de este mercado contempla la disputa por el recurso. A pesar de que en el interior de las aulas se promueve el trabajo articulado entre diversos profesionales, el mercado de consumo claramente funciona en dirección contraria. Aquí el concepto competencia, concepto muy usado en la actualidad educativa para hablar de proyectos curriculares, se refiere a dos ideas. Por un lado, a las habilidades que deben ser desarrolladas en los discentes por medio de las mallas curriculares $y$, por otro, a competir para captar la mayor cantidad de clientes posibles. Por consiguiente, aunque el proceso de acreditación se supone se estableció para proponer una revisión exhaustiva del funcionamiento universitario, su objetivo principal no va por este camino, "De este modo, tanto el licenciamiento como la acreditación, al adoptar sus mediciones a las características de las instituciones, han contribuido a validar el modelo de mercado (Rodríguez, 2012, p. 128).

\subsection{LA GLOBALIZACIÓN Y LA PÉRDIDA DE LA AUTONOMÍA UNIVERSITARIA}

En consecuencia, el modelo neoliberal de educación superior en Chile ha provocado la pérdida de la autonomía institucional, esto desde el punto de vista de las decisiones que se deban tomar como universidad en tanto institución que históricamente debe responder a los procesos de modernización de la sociedad y promover el conocimiento pertinente para sus propias realidades. En realidad, la autonomía queda relegada en tanto la universidad debe responder a cuestiones globales que además son vistas desde el prisma del modelo neoliberal, promovido y hegemonizado por la globalización y que en el ámbito de la investigación, innovación y vinculación queda subordinado a la indexación de las revistas o las publicaciones académicas, a la innovación global apuntalando procesos de reorientación organizacional para cumplir con estándares internacionales y las ha convertido en un bien de consumo para los investigadores que desean hacer carrera académica. Asimismo, para las universidades que desean mejorar en los rankings mundiales de calidad, este es el verdadero trampolín al éxito institucional, siempre y cuando se cumplan con los modelos de calidad requeridos. Esto de alguna manera puede producir efectos negativos en la identidad regional de las universidades, pues por tratar de emular modelos impuestos por medio de la globalización pierden el rumbo de su contexto local en el cual están insertos, ya que:

En un mundo mucho más móvil en el que el imaginario de los estudiantes considera la posibilidad de entrar a las universidades de la "super-liga", esto afecta no sólo la posición global de las universidades nacionales de investigación, sino también la solidez y legitimidad de esas universidades en sus propios países (Orodika, 2006, p. 40).

Esta racionalización impuesta por estándares internacionales de acreditación: impone, impacta y jerarquiza al sistema público y lo pondera en el sentido de las decisiones y las direcciones que debe tomar, por lo tanto, solo lo negociable, desde el 
punto de vista del mercado, es lo fundamentalmente necesario costear. "De esta manera lo educativo en su concepto tradicional queda reducido a lo financiable" (Donoso \& Alarcón, 2012, p. 38). Esto tiene una serie de implicancias tanto a nivel interno de las universidades como de quienes reciben sus servicios educativos, pues lo financiable está reducido al "eslogan" del éxito educativo, lo cual no es un invento, pues las carreras universitarias están supeditadas, socialmente, por jerarquizaciones de inversión, es decir, mientras más costosa sea la profesión, esta estará mejor ubicada en la jerarquización social, asimismo gozará de mayor prestigio, por lo tanto se transforma en un bien de consumo porque será una carrera más rentable cuando el egresado ejerza en el mundo laboral con su certificado obtenido. "En efecto, Benavente, Rapport y Meller muestran que en un conjunto de carreras universitarias hay diferencias de remuneraciones significativas dependiendo de la universidad en la cual obtuvo el título correspondiente" (Donoso \& Alarcón, 2012, p. 130).

Lo anterior es otro argumento más de lo neutralizada que se encuentra la universidad chilena con el modelo neoliberal impuesto por medio de la globalización, esto en términos que solo se ha reducido a ofrecer el éxito económico como oferta educativa (Bowles \& Gintis, 1981, p. 19) y no ha desarrollar en los estudiantes una formación profesional para responder a necesidades sociales, o que al menos ofrezca la oportunidad de discutir la pertinencia de su profesión en el entorno social. Al contrario, se moviliza netamente de acuerdo con la dirección que promueve el mercado imperante en la región. Por esta razón, se podría decir que las instituciones de educación superior están más orientadas en desarrollar una formación tecnocrática, por lo tanto, las competencias desarrolladas responderán una vez más a los requerimientos del mercado, es decir para mejorar la economía de unos pocos y no de la sociedad, sino de las transnacionales imperantes (Bürgi, 2017, p. 175).

\subsection{DISCURSO GLOBALIZADO POR MEDIO DE LAS UNIVERSIDADES}

Por este motivo, desde el punto de vista económico, para la educación superior, el mantener un discurso de "calidad", respecto de su oferta educativa, les permite mantener con vida no tan solo sus arcas financieras, sino también fomentar las esperanzas de la clientela que va surgiendo en torno a la promesa educativa que ofrecen y que es regulada por el mercado de consumo. Por ello las universidades en Chile se han orientado más en responder a cuestiones tecnocráticas que a asuntos con perspectivas sociales, y muchas de estas orientaciones están regidas por los resultados que obtienen en los procesos de acreditación, por lo tanto, las instituciones de educación superior solventan su supervivencia respondiendo a modelos centrados en realizar auditorías externas que focalizan su revisión en la solvencia económica de las carreras o de la institución en general, por ello nadie regula, ni siquiera el mercado, la cantidad abrumadora de estudiantes que se matriculan año a año en las diversas carreras que ofrecen las universidades, pues el tener un superávit, es un hermoso indicador de lo estable que es la economía universitaria que se sustenta en principios de equidad e igualdad, pero que solventa su capital gracias el hacinamiento en las salas de clases como modelo de ganancia. Lo anterior es herencia de la dictadura, que al parecer quienes definen la educación actual no quieren abandonar. En el caso de Chile, el modelo neoliberal con el 
regreso a la democracia no desapareció, se fortaleció, esto porque: "el Poder Ejecutivo desde el retorno a la democracia solo ha realizado ajustes menores al modelo de los años 80" (Rodríguez, 2012, p. 127).

Cambios irrelevantes que no han tenido la capacidad de redefinir en profundidad el rol de las universidades, y muchas de ellas aún en la actualidad están supeditadas a antiguos decretos impuestos en la dictadura, por ejemplo el del 30 de diciembre de 1980 artículo $1^{\circ}$ y $2^{\circ}$, "el cual se señala que la universidad debe atender a los intereses y necesidades del país al más alto nivel de excelencia" (Gajardo, 2005, p. 166), pero claro, esto lo fueron haciendo las instituciones de educación superior en un ambiente autoritario que no permitía que esas necesidades fueran establecidas en contra del ente que las regulaba, por lo tanto, fueron acomodándose a la filosofía del mercado imperante. Al respecto Donoso y Alarcón (2012) señalan: “Estas políticas respondieron a la transformación del rol del Estado desde una visión de benefactor a una subsidiaria, instalada en la dictadura cívico/militar desde casi los inicios de su gobierno (1975)" (p. 34). Dicho de otro modo, el Estado, perdió su rol orientador de políticas públicas educativas, ahora el que imponía estas normativas era la bancada privada, asimismo, su papel gubernamental que ejercía en las universidades fue traspasado al mercado imperante, el cual, "impone la racionalización y uso de los recursos que requieren los distintos financiamientos institucionales" (Gajardo, 2005, p. 166).

\section{CONCLUSIONES}

La clara adecuación del discurso y práctica universitaria chilena con un modelo neoliberal de educación universitaria, claramente expone las limitantes que tiene para que la universidad juegue el rol de ser espacio de debate y construcción del conocimiento pertinente para responder e incluso formular las preguntas que subyacen en el interior de esa sociedad que es mucho más compleja que las relaciones de oferta y demanda del mercado. La ventaja que aporta el modelo de gestión neoliberal es la "justificación" de unos límites que no sólo quedan superados por las complejas realidades sociales, culturales y políticas de la sociedad chilena (una más de las sociedades latinoamericanas en construcción). El que esto sea así, permite no sólo mostrar que esos límites propios del modelo se asumen como pertinentes para una realidad que deja por fuera una de las tareas fundamentales de la universidad como espacio de construcción del conocimiento. Sólo la construcción de un conocimiento "productivo" adecuado al discurso hegemónico globalizador, es insuficiente cuando las múltiples dimensiones y complejidades de una sociedad multicultural, con amplios sectores aún en construcción de sus propios espacios de indagación sobre el rol en la sociedad están en construcción.

En este trabajo, ha predominado la consideración de la educación universitaria sin desplegar lo que se supone será un trabajo posterior sobre los retos que se impone en la producción de conocimiento e innovación propias de cada sociedad. Al hacerlo así, se hace evidente lo que es fundamental destacar: la universidad al producir un sujeto/producto en el proceso educativo universitario va construyendo una identidad que está centrada en la idea de consumo (punto de encuentro entre oferta y demanda) y que condiciona a todo el aparato institucional a realizar el acto de la producción universitaria como un acto de consumo. El que esto ocurra de este modo, precisamente pone en entredicho la más esencial condición del ser humano en lo que concierne al conocimiento: la posibilidad de preguntarse, por aquello que precisamente no se puede 
consumir: el afán de trascender del presente, esa aparente condición final sobre la cual el neoliberalismo se erige e impone a nivel global.

Los recientes sucesos en torno a las manifestaciones sociales en Chile y la insurgencia de nuevos actores guiados por una racionalidad con mayor ambición en el tiempo, parecen demostrar que la naturaleza del ser humano se resiste a ser confinada en la noción de consumo inmediato. Sobre este aspecto, quizás la globalización logra imponer un presente denso (en términos de consumo) que oculta el futuro que luce poco probable para las marginales mayorías que habitan el continente latinoamericano.

Artigo recebido em: $31 / 01 / 2020$

Aprovado para publicação em: 11/04/2020

\section{THE INSTITUCIÓN UNIVERSITARIA EN LA GLOBALIZACIÓN NEOLIBERAL: CONSIDERATIONS FROM A PERSPECTIVE OF LA GESTIÓN UNIVERSITARIA}

ABSTRACT: A neoliberal-inspired university management model, typical of the globalizing hegemonic discourse, is presented to account for the process of building the Chilean university of the present. The university management model rests on the three axes considered strategic in the contemporary university and privileges management from a neoliberal rationality that underlies organizational processes, in particular evaluation and accreditation.

KEYWORDS: Rationality. Neoliberalism. College. Chile.

A INSTITUIÇÃO UNIVERSITÁRIA NA GLOBALIZAÇÃO NEOLIBERAL: CONSIDERAÇÕES DE UMA PERSPECTIVA DE GESTÃO UNIVERSITÁRIA

RESUMO: Um modelo de gestão universitária de inspiração neoliberal, típico do discurso hegemônico globalizante, é apresentado para dar conta do processo de construção da universidade chilena na atualidade. O modelo de gestão universitária assenta nos três eixos considerados estratégicos na universidade contemporânea e privilegia a gestão a partir de uma racionalidade neoliberal subjacente aos processos organizacionais, em particular a avaliação e a acreditação.

PALABRAS-CHAVE: Racionalidade. Neoliberalismo. Universidade. Chile

\section{REFERENCIAS}

BASSO, Patricio. Educación superior en Chile: El fracaso de un modelo neoliberal. Límite. Revista interdisciplinaria de filosofía y psicología, 11 (37), 21, 48, 2016.

BOWLES, Samuel, \& GINTIS, Herbert. La instrucción escolar en la américa capitalista. Bogotá: Siglo Veintiuno, 1981. 
BROWN, Wendy. Neoliberalism and the End of Liberal Democracy. Edgework: Critical Essays on Knowledge and Politics. 2005.

BÜRGI, Regula. El mundo libre y el culto al experto: el auge de la tecnocracia educacionalizadora de la OCDE. Profesorado. Revista de Curriculum y formación del profesorado, 21 (2), 159-181, 2017.

CARVAJAL, José. Universidad y globalización: cambios y desafíos. Praxis \& Saber, 8 (18), 149-167, 2017.

CASANOVA, Geo Saura; BOTíA, Antonio Bolívar. Sujeto Académico Neoliberal: Cuantificado, Digitalizado y Bibliometrificado. REICE: Revista lberoamericana sobre Calidad, Eficacia y Cambio en Educación. vol. 17, no 4, p. 9-26, 2019.

DONOSO, Sebastián y ALARCON, Jorge. El lucro en la educación chilena: debate conceptual acerca del sentido de la educación pública y de la privada. Pro-Posicoes, 23 (2), 33-49, 2012.

FUENMAYOR, Ramsés., BONUCCl, Mario, \& LÓPEZ-GARAY, Hernán. An interpretivesystemic study of the University of Los Andes. Systems practice, 4(5), 507-525, 1991.

GAJARDO, Tatiana. Educación privada V/S educación pública en Santiago de Chile: una reflexión necesaria. En Espacio público y privado del conocimiento. Estudios sobre políticas universitarias en América Latina, Clacso, 163-203. 2005.

HABERMAS, Jürgen. Legitimation crisis. Beacon Press. 1975

HABERMAS, Jürgen. On the logic of the social sciences. MIT Press. 1990

IBARRA, Eduardo. Privatización y comercialización de la universidad. Las disputas del conocimiento. Revista Perfiles Educativos, XXXIV, 2012.

MACINTYRE, Alasdair. God, Philosophy, Universities. A history of the Catholic Philosophical Tradition. Londres, 2009.

MEJÍA JIMNEZ, Marco. Educación(es) en la(s) globalización(es). Entre el pensamiento único y la nueva crítica. Bogotá: Desde abajo, 2011.

ORDORIKA, Imanol. Educación superior y globalización: las universidades públicas frente a una hegemonía. Andamios, 3 (5), 31-47, 2006.

PRESSACO, Carlos \& CARBONE, Ricardo. Educación superior en Chile: tensiones en torno al eje de calidad-equidad. Revista Papel Político, 15 (2), 537-570, 2010. 
RODRIGUEZ, Emilio. La educación superior en Chile y el rol del mercado: ¿Culpable o inocente? ingeniare. Revista Chilena de Ingeniería, 20 (1), 126-135, 2012.

TORRES, Carlos \& SCHUGURENSKY, Daniel. The political economy of higher education in the era of neoliberal globalization: Latin America in comparative perspective. Higher Education, 43(4), 429-455, 2002.

\footnotetext{
Alejandro Elas OchoA.ArIas: Catedrático de la Universidad de Los Andes, Venezuela e Investigador Senior en Systemología Interpretativa y Director del Centro de Investigaciones en Sistemología Interpretativa de la Universidad de Los Andes. Obtuvo su maestría en Gestión de la Información en la Universidad de Lancaster (Reino Unido) y su doctorado en la Universidad de Hull (Reino Unido). Investigador invitado en la Universidad de Hull (Reino Unido) y la Universidad de Cuyo (Mendoza, Argentina).

Orcid: https://orcid.org/0000-0001-8464-7108

E-mail: dioseses@gmail.com
}

Jesús Lara-Coronado: Doctor en Pedagogía por la Universidad Nacional Autónoma de México. Posee una Maestría en Educación por la Universidad de Santiago de Chile. Es profesor de tiempo completo de la Escuela de Pedagogía en Educación Básica de la Universidad Austral de Chile, sede Puerto Montt. Sus líneas de investigación se han centrado en la historia de la educación por medio del teatro y el análisis histórico de la influencia educativa de los dominicos en Chile.

Orcid: https://orcid.org/0000-0003-0365-4598

E-mail: jesus.lara@uach.cl

Este periódico utiliza a licença Creative Commons Attribution 3.0, para periódicos de acesso aberto (Open Archives Iniciative - OAI) 